\title{
Transobturatoric tape procedure for female stress urinary incontinence
}

\author{
Titta Joutsiniemi • Seija Ala-Nissilä • Raija Räty • \\ Eija Laurikainen $\cdot$ Pentti Kiilholma
}

Received: 28 May 2008 / Accepted: 27 June 2008 / Published online: 6 August 2008

(C) Springer-Verlag 2008

\begin{abstract}
To assess the efficacy and safety of the transobturatoric tape (TOT) procedure as a treatment for female stress urinary incontinence (SUI). All patients $(n=191)$ who underwent TOT between May 2003 and December 2004 were studied retrospectively. The study protocol involved recording of preoperative and perioperative details and postoperative subjective evaluation. The subjective outcome was assessed with two questionnaires at a mean of 20 and 34 months after the operation. The mean operation time of the patients without concomitant procedure was $19 \mathrm{~min}$. The complication rate was low and postoperative recovery quick. The follow-up visit was scheduled $2-3$ months after the procedure. The objective outcome assessment was done at the hospital for $50 \%$ of the patients, and the other half of the patients were followed up by their own gynecologist. During the follow-up visit ( $n=151), 79 \%$ of the women were cured: $90 \%$ of the patients with genuine SUI and $60 \%$ of those with mixed urinary incontinence (MUI). The data of 40 patients was either not available from patients' private gynecologists or the patients had not undergone a follow-up visit at all. For the first subjective outcome assessment, participating patients $(n=188)$ received a questionnaire. Three patients had died from unrelated causes during this follow-up. The response rate was $82.4 \%$. Overall, $83.9 \%$ of the respondents reported significant improvement, of which $90.5 \%$ of the patients had genuine SUI and $70.0 \%$ of the patients had MUI, respectively. During the second outcome assessment, the response rate was $85.1 \%(160 / 188)$. At a
\end{abstract}

T. Joutsiniemi $(\varangle) \cdot \mathrm{S}$. Ala-Nissilä $\cdot$ R. Räty $\cdot$ E. Laurikainen

P. Kiilholma

Department of Obstetrics and Gynecology,

Turku University Hospital,

Finland Kiinamyllynkatu 4-8,

20520 Turku, Finland

e-mail: titta.joutsiniemi@tyks.fi mean of 34 months postoperatively, $87.5 \%$ of the patients reported significant improvement: $94.5 \%$ of the patients with genuine SUI and $72.0 \%$ of those with MUI. The incontinence operation through the transobturatoric route is a safe and effective treatment, especially for patients with pure SUI. The rate of continence was sustained during a follow-up of 34 months.

Keywords Stress urinary incontinence .

Subjective outcome $\cdot$ Transobturatoric tape procedure

$\begin{array}{ll}\text { Abbreviations } \\ \text { TOT } & \text { transobturatoric tape } \\ \text { SUI } & \text { stress urinary incontinence } \\ \text { MUI } & \text { mixed urinary incontinence } \\ \text { TVT } & \text { tension-free vaginal tape } \\ \text { TVT-O } & \text { transobturator vaginal tape (inside-out) } \\ \text { UISS } & \text { Urinary Incontinence Severity score } \\ \text { DIS } & \text { Detrusor Instability Scores } \\ \text { VAS } & \text { Visual Analogue Scale } \\ \text { UDS } & \text { urodynamic study }\end{array}$

\section{Introduction}

Minimal invasive procedures have become popular for the treatment of female stress urinary incontinence (SUI) since 1995 [1]. Tension-free vaginal tape (TVT), which has become a golden standard among the operations for SUI, is based on the theory that the female urethra is supported by its mid-portion [2]. It has been proven to be an effective surgical procedure: Cure rates have varied from $85 \%$ up to $100 \%$ [3-5] and TVT seems also to fulfill the expectations of high cure rates in follow-up assessments lasting more than 5 years [6]. 
In 2001, Delorme [7] described a new method for the treatment of female SUI, the tension-free transobturator tape (TOT) procedure, in which the tape is placed through the obturator foramina. The new technique has become popular in Finland since 2003. Trials comparing TVT and TOT have shown that TOT seems to be a safe and effective method and is associated with less major hemorrhages and bladder and bowel perforations than TVT, with 1 year cure rates of $90-95 \%$ [8-10]. The aim of the present study was to report the outcome of 191 patients who underwent TOT procedure in one Finnish university clinic.

\section{Materials and methods}

A retrospective study of all patients $(n=191)$ who underwent outside-in TOT procedure (Monarc $\left.{ }^{\circledR}\right)$ at the Turku University Hospital between May 2003 and December 2004 was carried out. Preoperatively, urogynecologic histories were obtained, urine samples were analyzed, and physical examinations were done, with particular attention to hypermobility of the urethrovesical junction. The study protocol involved recording of preoperative and perioperative details and postoperative subjective evaluation. Incontinence symptoms were assessed using validated Urinary Incontinence Severity Score (UISS) and Detrusor Instability Score (DIS) questionnaires pre- and postoperatively [11]. Visual Analogue Scale (VAS), in which 0 means no urinary symptoms and 100 means the worst possible urinary symptoms, was recorded and patient's satisfaction with the outcome was assessed after the operation.

A supine stress test with a mean of 300-ml bladder volume was performed. Patients with symptoms typical of SUI, positive stress test results, and bladder neck hypermobility underwent operation without a prior urodynamic study (UDS). Instead, preoperative UDS were made in 57 patients in whom a history and results of the clinical examination conflicted with diagnosis of genuine SUI. In 14 patients $(7.3 \%)$, a urogynecologial ultrasonography was done. In the study population, $66 \%$ of the women were diagnosed to have SUI and 34\% had MUI using the DIS.

The TOT procedure was performed according to the outside-in technique described by Delorme [8]. A urinary catheter was inserted before the procedure and was removed immediately after the procedure in patients without concomitant surgery. A vaginal incision of $2 \mathrm{~cm}$ was made at a point $1 \mathrm{~cm}$ below the urethral meatus. Dissection of the paraurethral spaces was made laterally toward the ischiopubic ramus on both sides. A $0.5-\mathrm{cm}$ vertical skin incision was made on the internal surface of the thigh on both sides. The needle of the device was introduced through the skin incision and forwarded to the vaginal incision, while the operator's protecting finger remained in contact with the needle protecting the urethra. Surgical tools were used, and a synthetic tape is passed underneath the urethra through the obturator foramina toward the thigh folds. The tape was placed in a transverse plane between the urethra and vaginal wall without any tension. Peroperatively, no routine cystoscopy or cough test was done.

Antibiotic prophylaxis (500 mg metronidazole) was given to all patients. Thrombosis prophylaxis was given during the hospital stay. Residual urine volume was measured after the first spontaneous micturition 3-4 h postoperatively or after urinary catheter removal the next morning. Another measurement was carried out if the first residual volume was $>100 \mathrm{ml}$. Women with residual urine volumes repeatedly more than $100 \mathrm{ml}$ were primarily treated with cholinergic medication. In some cases, intermittent self-catheterization was performed or an indwelling catheter was used.

Because of a novel operation, the first TOT being performed in May 2003 in our hospital, a follow-up visit was scheduled for 2 to 3 months after the operation at the study hospital in the beginning of the study period $(n=96)$. Later on, the follow-up visit was done by the patient's private gynecologist. Overall, we were able to collect details of the follow-up visit from $79 \%$ of the patients $(n=151)$.

The subjective outcome was assessed with two questionnaires at a mean of 20 (range, 13-32) and 34 (range, 16-48) months after the operation. The first assessment of all participating patients was made with a non-validated postal questionnaire of subjective evaluation of continence (Appendix 1), UISS, DIS, and VAS. A total of $82.4 \%$ (155/188) of the patients answered the questionnaires. Three patients had died of unrelated causes during a follow-up period. For the second outcome assessment, we got a subjective outcome from $85.1 \%$ of the patients $(160 / 188)$. Patients $(n=18)$ who reported significant dissatisfaction (UISS $>50$ ) were offered a charge-free follow-up visit to the hospital. Chi-square test and $t$ test procedure were used for statistical analysis.

The study was approved by the Ethics Committee of the hospital.

\section{Results}

The basic characteristics of the patients are presented in Table 1. Forty-five percent $(n=85)$ of the patients underwent concomitant surgery: prolapse surgery in 58 patients (anterior and/or posterior colporraphy or sacrospinous fixation) and vaginal or laparoscopic hysterectomy in 37 patients. The procedures were performed under spinal $(52 \%)$, general $(47 \%)$, or local $(1 \%)$ anesthesia. The mean operation time of the patients without concomitant proce- 
Table 1 Patient characteristics

\begin{tabular}{ll}
\hline Characteristics & Values \\
\hline Number of patients (N) & 191 \\
Age (years) & $59(31-86)$ \\
Parity & $2.0(0-5)$ \\
BMI (body mass index) & $27.3(19.1-47.0)$ \\
No concomitant disease & $91 / 47.6 \%$ \\
Hormonal Therapy & $81 / 42.4 \%$ \\
Previous gynecological operations & $125 / 65.4 \%$ \\
Previous incontinence operations & $32 / 16.8 \%$ \\
\hline
\end{tabular}

Values are given as median (range) or number and percent.

dure was $19 \mathrm{~min}$ (range, 6-45 $\mathrm{min}$ ). Mean blood loss related to transobturator procedures was minimal and was not recorded separately.

There were two urethral perforations $(1.0 \%)$ during the Monarc ${ }^{\circledR}$ procedure. In one patient, it was noticed during the procedure because of hematuria. Subsequent urethrocystoscopy revealed urethral perforation. The device was pulled back and guided more laterally. Patient's recovery was uneventful, and she was continent postoperatively. The other patient presented macroscopic hematuria on the first postoperative day, and urethral perforation was diagnosed by cystoscopy. The tape was seen passing through the urethra. The tape was cut and the vaginal epithelium was covered over surgically. The patient had an indwelling catheter for 3 days and a suprapubic catheter for 1 week. Afterwards, she was incontinent and was reoperated successfully by transobturator vaginal tape inside-out (TVT-O) procedure after 3 months. The vaginal skin perforation was the most common perioperative complication $(11.2 \%)$. There were two patients who had secondary exposure of the tape at the postoperative follow-up. They were reoperated without further sequelae: In one patient, the tape was cut and covered with vaginal epithelium, and in another patient, only the tape was covered. There were no tape displacements. Neither heavy bleeding nor postoperative hematomas were reported. Three patients $(1.6 \%)$ had postoperative urinary infection before discharging; no other infections were diagnosed during the hospital stay. There were no perforations of the bladder, blood vessels, or the bowel.

The first postoperative residual urine volume among patients without concomitant surgery was a mean of $202 \mathrm{ml}$ (range, 2-900). Two patients (1.9\%) needed a urinary catheter overnight. Among patients with concomitant surgery, the first urine residual volume was $132 \mathrm{ml}$ (range, $2-700)$. Five percent $(n=10)$ of these patients experienced temporary urinary retention. Two patients performed intermittent self-catheterization, one patient had an indwelling catheter for 1 month, and one patient underwent urethral dilation. All these problems were revealed at the first follow-up visit. None of the patients experienced postoperative groin pain.

Fifty percent of the patients $(n=96)$ were followed by the institution within 2 to 3 months postoperatively; other patients went to the follow-up visit with their private gynecologist. The data concerning the follow-up visit was available for 151 patients (79\%). Seventy-nine percent of these patients were completely cured $(n=119)$ and $11 \%$ were markedly improved $(n=16)$. Among the patients with SUI, 96\% was completely cured or markedly improved and $78 \%$ among those with MUI (Fig. 1). De novo urge symptoms were reported in $5.5 \%$, and occasional voiding difficulties were complained by $5.5 \%$ of patients $2-$ 3 months after the operation.

The first questionnaire including subjective evaluation of continence (Appendix 1) was sent to the patients twice postoperatively. The patients were divided into three groups according their answers: completely satisfied, satisfied, and not satisfied. The patients who were completely satisfied were subjectively dry, the satisfied patients reported significant improvement of their continence, and those not satisfied still experienced some symptoms of SUI or de novo urge. During a mean follow-up time of 20 months (range, 13-32) three patients were deceased. After one reminder, a total of $82.4 \%(155 / 188)$ of the patients returned the questionnaires. Of the respondents, $83.9 \%$ were completely dry or markedly improved. Among the patients with genuine SUI, the respective rate was $90.5 \%$ and $70.0 \%$ among patients with MUI (Fig. 2). Pre- and postoperative DIS and UISS and postoperative VAS are presented in Table 2. The data of the patients with concomitant surgery were separately analyzed and presented in Table 3. De novo urge symptoms were reported in

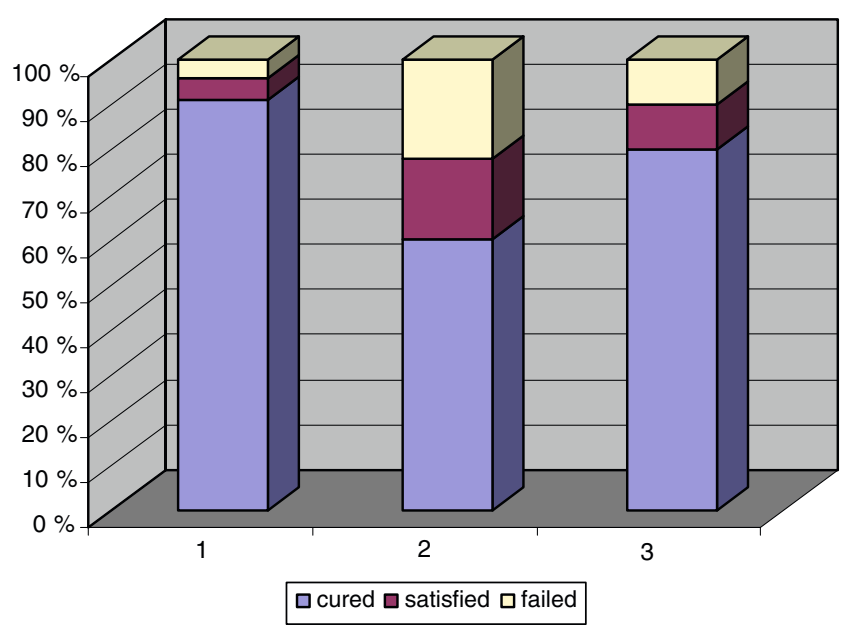

Fig. 1 Results at the first follow-up visit 2-3 months postoperatively $(n=151)$. 1 SUI, 2 MUI, 3 total, $p<0.0001$ between SUI and MUI 


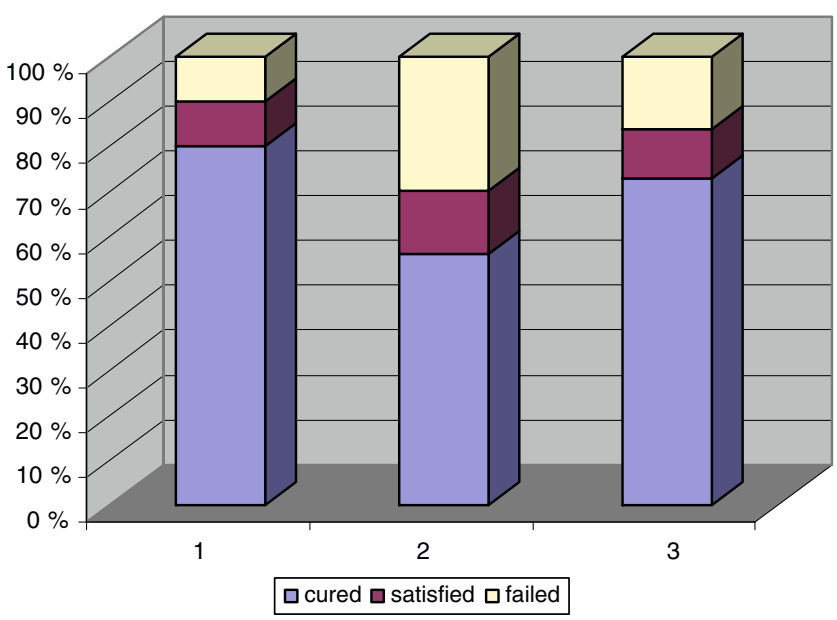

Fig. 2 Subjective outcome at the mean of 20 months postoperatively $(n=155)$. 1 SUI, 2 MUI, 3 total, $p<0.005$ between SUI and MUI

$15.4 \%$ of the patients and occasional voiding difficulties in $3.9 \%$.

The second evaluation was performed a mean of 34 months (range 16-48) after the operation with a mailed questionnaire. We got subjective outcome assessments from $85.1 \%$ of the patients $(160 / 188)$. Of the respondents, $94.5 \%$ of the patients with genuine SUI and $81.8 \%$ of the patients with MUI were completely cured or significantly improved (Fig. 3). Although de novo urge symptoms were reported by $21.6 \%$ of the respondents, anticholinergic medication was needed only by $2.7 \%$ of the patients. Occasional voiding dysfunction was reported by $3.8 \%$ of the respondents. Three patients $(1.6 \%)$ had suffered from recurrent lower urinary tract infections.

At the second evaluation, significant dissatisfaction with the operation was reported by 18 patients. One of these patients had already undergone TVT procedure previously. Seven patients underwent preoperative UDS revealing SUI, while other patients had a positive stress test. An offered charge-free follow-up visit to the hospital was attended by 15 patients. The main complaint was urgency symptoms $(n=10)$. Seven patients were treated with anticholinergic medication. All patients were taught pelvic floor muscle training, and five patients were sent to a physiotherapist for electromyography-biofeedback assisted training. Four patients reported better continence postoperatively and therefore experienced no need for further treatment. One patient needed an operation for recurrent SUI. During this follow-up of a mean of 34 months, altogether, six patients were reoperated for recurrence.

\section{Discussion}

The surgery of SUI has dramatically changed during the last 10 years. Open colposuspension was first described by Burch in 1961 [12]. Nowadays, minimally invasive procedures have replaced this procedure as the primary surgical treatment for incontinence. In a survey performed by the International Urogynecology Association regarding the practice in the surgical management of SUI, the preferred primary anti-incontinence procedure was TVT (68\%) followed by TOT and open or laparoscopic colposuspension (13\% each) [13].

In our study, two urethral perforations (1.1\%) were observed. There were no bowel, vascular, or nerve injury nor bladder perforations. In an earlier study of 183 patients with SUI undergoing TOT, the overall complication rate was $2.2 \%$ (one bladder perforation, two urethral perforations, and one lateral vaginal perforation) [14]. In a study of 604 TOT patients, the reported incidence of bladder perforation was $0.5 \%$, vaginal perforation $0.33 \%$, hemorrhage $0.83 \%$, and hematoma $0.33 \%$ [15]. Fischer et al. [9] also reported a very low rate of complications and adverse events of the transobturatoric approach, and they preferred this procedure over TVT.

The most common complication in our study was vaginal mucosal perforation. Most of these complications were related to procedures performed by less-experienced surgeons. In our study, operations were performed by a heterogeneous group of physicians, including urogynecologists, gynecologists, and junior physicians operating under the guidance of a senior. These series include each surgeon's learning curve. Obviously a short learning curve in the absence of complications with long-term adverse sequelae enhances the feasibility and safety of the transobturator technique even more [15].

Table 2 Pre- and postoperative Detrusol Instability Scores (DIS) and Urinary Incontinence Severity Scores (UISS) and postoperative Visual Analogue Scale at the mean follow-up of 20 months

\begin{tabular}{lccccc}
\hline & $\begin{array}{l}\text { Preoperative DIS, } \\
n=114\end{array}$ & \multicolumn{2}{c}{$\begin{array}{l}\text { Postoperative DIS, } \\
n=142\end{array}$} & $\begin{array}{l}\text { Preoperative UISS, } \\
n=113\end{array}$ & $\begin{array}{l}\text { Postoperative UISS, } \\
n=141\end{array}$ \\
\hline SUI & 6.2 & 4.1 & 52.7 & 12.2 & $\begin{array}{l}\text { Postoperative VAS, } \\
n=145\end{array}$ \\
MUI & 9.7 & 7.4 & 69.6 & 35.4 & 39 \\
\hline
\end{tabular}

VAS was not recorded preoperatively. There was a statistically significant difference between preoperative DIS and postoperative DIS $(p<0.0001)$ and between preoperative UISS and postoperative UISS $(p<0.0001)$.

SUI Stress urinary incontinence, MUI mixed urinary incontinence 
Table 3 Patients undergoing TOT operations alone or with concomitant procedure

Only TOT operations, $n=106$

$1.9 \%(n=2)$
$202 \mathrm{ml}(2-900)$
$0.7(0-4)$
$72.1 * / 81.4^{*}$
$67.0^{* * / 83.0 * *}$

TOT with concomitant procedure, $n=85$

$$
\begin{aligned}
& 78.8 \%(n=67) \\
& 132 \mathrm{ml}(2-700) \\
& 2.4(0-12) \\
& 73.9 * / 87.0^{*} \\
& 70.0^{* * / 92.7 * *}
\end{aligned}
$$

There was no statistically significant difference between TOT operations with or without concomitant procedure ( $p=0.538$ at the first subjective evaluation and $p=0.163$ at the second subjective evaluation)

Urinary incontinence has an influence on quality of life and self-confidence. Recently, subjective outcome measurements have also been included in the postoperative evaluation of incontinence surgery [16-19]. In one study [19], the subjective cure rate was reported to be significantly greater than the objective cure rate. In another study, there were no differences in objective and subjective cure rates [20]. In our study, those 18 patients who reported symptoms of urinary incontinence or dissatisfaction with the operation were offered a charge-free visit at the hospital. The main reason for dissatisfaction was found to be urgency symptoms, which were complained by ten patients. Only one patient needed reoperation for SUI.

Two multicenter studies confirmed the cure rate over $84 \%$ at 3 months follow-up after TOT in patients with SUI $[14,20]$. In another retrospective multicenter study, the satisfaction rate of $85.5 \%$ was reported after 1-year followup of 131 patients [15]. After a mean follow-up of 2 years, subjective and objective continence rates of $92 \%$ and $80 \%$ were observed in a study of Giberty et al. [19]. Similarly, in our study, more than $83 \%$ of the patients reported significant subjective improvement of continence at 20 and 34 months postoperatively.

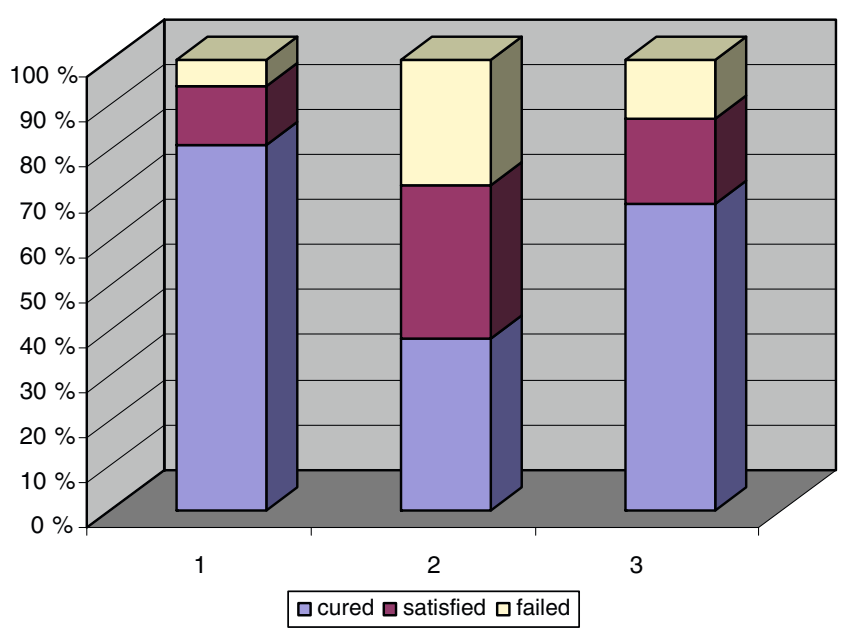

Fig. 3 Subjective outcome at the mean of 34 months postoperatively $(n=160)$. 1 SUI, 2 MUI, 3 total, $p<0.0001$ between SUI and MUI
In the present study, there were few postoperative complications, and the majority of the patients without concomitant surgery were discharged on the day of the operation or on the first postoperative day. A relatively high incidence of de novo urgency was observed, although anticholinergic medication was needed only in a few patients. In previous studies, the incidence of de novo urge incontinence has varied from $2 \%$ to $15 \%[10,19]$. In Krauth's study [15], transient postoperative retention was found in $1.5 \%$ of the patients, and $3 \%$ of non-satisfied patients required repeat incontinence surgery. In the followup visit 2-3 months postoperatively, there were no major retention problems, although some difficulties to empty the bladder was reported by $5.2 \%$ and $4.5 \%$ of the patients at later follow-up after 20 and 34 months. No cases of dysuria, pelvic pain, or dyspareunia were reported. Only $3 \%$ of our patients needed reoperation for SUI.

In an earlier Finnish study, the cure rates after incontinence surgery (TVT) among patients with SUI were 97\% and $69 \%$ among patients with MUI [21]. Furthermore, a resent study confirmed the cure rate after TVT procedure better in women with predominant stress incontinence and those with stress and urge incontinence equally than those with predominant urge incontinence [22]. Our study confirms that the success rate is considerably better in patients who undergo TOT for genuine SUI than for MUI. In addition, the recurrence of incontinence seems to be more common in patients with MUI than with SUI during a follow-up period. The study presented in this paper suggests that TOT procedure might be done effectively with concomitant vaginal surgery. In a previous study comparing TVT with or without concomitant procedure, more complications were reported after TVT with concomitant surgery than after TVT alone, but cure rates did not differ statistically significantly [23]. In addition, Mellier et al. [17] concluded that pelvic floor defects, benign uterine disorders, and SUI can be safely treated concomitantly using vaginal procedures and TOT.

This retrospective study reports mainly a subjective evaluation of outcome after TOT procedure. The response rates to the questionnaires were quite high during a mean follow-up of 34 months. Randomized, prospective trials are 
needed to confirm a long-term efficacy of these operations especially in comparison with other mini-invasive antiincontinence procedures.

\section{Appendix 1: Questionnaire for assessing subjective outcome}

1. Have you had urinary infections following the operation?

No/Yes, how many

2. Have you experienced a frequent need to urinate?

No/Yes,

As often as/more often than preoperatively

A medication for this symptom:

3. Have you experienced difficulty in emptying the bladder?

No/Yes

4. Have you had any operative treatment for urinary symptoms following the operation?

No/Yes

What kind of procedure:

5. Have you experienced leakage of urine related to physical activity?

No/Yes,

As often as/more often than preoperative

6. Have you experienced involuntary leakage of urine following the operation?

No/Yes

7. Do you need pads?

No/Yes

How many pads per day:
8. Are you satisfied with the operation?

Completely satisfied/Satisfied

Not satisfied, why?

9. Would you recommend this operation to yours friends?

Yes/No

\section{References}

1. Ulmsten U, Petros P (1995) Intravaginal slingplasty (IVS): an ambulatory surgical procedure for treatment of female urinary incontinence. Scand J Urol Nephrol 29:75-82

2. Petros P, Ulmsten U (1993) An integral theory and its method for the diagnosis and management of female urinary incontinence. Scand J Urol Nephrol Suppl 153:1-93

3. Ulmsten U, Henriksson L, Johnson P, Varhos G (1996) An ambulatory surgical procedure under local anesthesia for treatment of female urinary incontinence. Int urogynecol J 7:81-86

4. Nilsson CG (1998) The tensionfree vaginal tape procedure (TVT) for treatment of female urinary incontinence. A minimal invasive surgical procedure. Acta Obstet Gynecol Scand Suppl 168:34-37

5. Ulmsten U, Falconer C, Johnson P et al (1998) A multicenter study of tension-free vaginal tape (TVT) for surgical treatment of stress urinary incontinence. Int Urogynecol J 9:210-213

6. Nilsson CG, Falconer C, Rezapour M (2004) Seven-year followup of the tension-free vaginal tape procedure for treatment of urinary incontinence. Obstet Gynecol 104:1259-62

7. Delorme E (2001) Transobturator urethral suspension: miniinvasive procedure in the treatment of urinary stress incontinence in women. Prog Urol 11:1306-1313

8. Mellier C, Benayed B, Bretones S, Pasquier JC (2004) Suburethral tape via the obturator route: is the TOT a simplification of the TVT? Int Urogyn J 15:227-232

9. Fischer A, Fink T, Zachmann S, Eickenbusch U (2005) Comparison of retropubic and outside-in transobturator sling systems for the cure of female genuine stress urinary incontinence. Eur Urol 48:799-804

10. Juma S, Brito C (2007) Transobturator Tape (TOT): two years follow-up. Neurourol Urodynam 26:37-41

11. Stach-Lempinen B, Kujansuu E, Laippala P, Metsänoja R (2001) Visual analoque scale, urinary incontinent severity score and 15 D-psychometric testing of three different health-related quality-oflife instruments for urinary incontinent women. Scand J Urol Nephrol 35:476-483

12. Burch JC (1968) Cooper's ligament urethrovesical suspension for stress incontinence. 3 years experience results, complications, technique. Am J Obstet Gynaecol 100:764-74

13. Jha S, Arunkalaivanan AS, Davis J (2005) Surgical management of stress urinary incontinence a questionnaire based survey. Eur Urol 47:648-652

14. Costa P, Grise P, Droupy S et al (2004) Surgical treatment of female stress urinary incontinence with a trans-obturator-tape (T. O.T.) Uratape: Short term results of a prospective multicentric study. Eur Urol 46:102-107

15. Krauth JS, Rasoamiaramanana H, Barletta H et al (2005) Suburethral tape treatment of female urinary incontinence - morbidity 
assessment of the trans-obturator route and a Newtape (1-STOP): A multi-centre experiment involving 604 cases. Eur Urol 47:102-107

16. Deval B, Ferchaux J, Berry R et al (2006) Objective and subjective cure rates of transobturator tape (Ob-Tape) treatment of female urinary incontinence. Eur Urol 49:373-377

17. Mellier G, Mistrangelo E, Gery L et al (2006) Tension-free obturator tape (Monarc subfascial hammock) in patients with or without associated procedures. Int Urogynecol J Pelvic Floor Dysfunct 14:225-232

18. Romeguere T, Quackels T, Bollens R et al (2005) Transobturator vaginal tape (TOT) for female stress urinary incontinence: one year of follow up in 120 patients. Eur Urol 48:805-809

19. Giberty C, Gallo F, Cortese P, Schenone M (2007) Transobturator tape for treatment of female stress urinary incontinence: objective and subjective results after a mean follow-up of two years. Urology 69:703-707
20. Barry C, Lim Y, Muller R et al (2007) A multi-centre randomised clinical control trial comparing the retropubic (RP) approach versus the transobturator approach (TO) for tensionfree, suburethral sling treatment of urodynamic stress incontinence: the TORP study. Int Urogynecol J Pelvic Floor Dysfunct 19:171-178

21. Laurikainen E, Kiilholma P (2003) The tension-free vaginal tape procedure for female urinary incontinence without preoperative urodynamic evaluation. J Am Coll Surg 4:579-583

22. Kulseng-Hanssen S, Hysby H, Schiøtz A (2008) Follow-up of TVT operations in 1113 women with mixed urinary incontinence at 7 and 38 months. Int Urogynecol J 19:391-396

23. Meltomaa S, Backman T, Haarala M (2004) Concomitant vaginal surgery did not affect outcome of the tension-free vaginal tape operation during a prospective 3-year follow up study. J Urol $172: 222-226$ 